\title{
Loss of convexity and embeddedness for geometric evolution equations of higher order
}

\author{
SIMON BLATT
}

Abstract. We show that for a large class of geometric evolution equations of immersed surfaces in the Euclidean space, there are compact embedded surfaces that lose their embeddedness and compact strictly convex surfaces that lose their convexity under these evolution equations.

\section{Introduction}

For many geometric evolution equations of second order such as the curve shrinking flow and the mean curvature flow, the parabolic maximum principle implies that embedded curves stay embedded under this flow and convex curves stay convex and even shrink to round points (cf. [1-3]). However, there is evidence that geometric evolution equations of higher order do not share these properties. For the surface diffusion flow there are analytic proofs that neither embeddedness nor convexity is preserved under the flow (cf. $[4,5]$ ) and for the surface diffusion equation of curves we know that strictly convex curves can become nonconvex [6]. For the Willmore flow, loss of embeddedness is proven in [7], while numerical experiments indicate that convexity can be lost as well [8].

These results are special cases of a general theorem we prove in this short note. We consider smooth families of immersions $f_{t}: M_{n} \rightarrow \mathbb{R}^{n+1}, t \in[0, T)$, of an $n$-dimensional, orientable manifold $M_{n}$ without boundary that satisfy a geometric evolution equation of the form

$$
\partial_{t} f_{t}=\left(\left(-\Delta_{f_{t}}\right)^{p} H_{f_{t}}+B\left(\nabla_{f_{t}}^{2 p-1} S_{f_{t}}, \nabla_{f_{t}}^{2 p-2} S_{f_{t}}, \ldots, S_{f_{t}}, v_{f_{t}}\right)\right) v_{f_{t}}
$$

Here, $v_{f_{t}}$ denotes the unit normal along $f_{t}, \Delta_{f_{t}}$ the Laplace-Beltrami operator, $S_{f_{t}}:=$ $-D v_{f_{t}}$ the shape operator, $H_{f_{t}}=\operatorname{tr}(S)$ the mean curvature, $\nabla_{f_{t}}$ the covariant derivative along $f_{t}$, and $B$ is a smooth function on

$$
\left(\left(T M_{n}^{*}\right)^{2 p} \otimes T M_{n}\right) \times\left(\left(T M_{n}^{*}\right)^{2 p-1} \times T M_{n}\right) \times \cdots \times\left(T M_{n}^{*} \otimes T M_{n}\right) \times \mathbb{R}^{n+1} .
$$


Then, the following statement holds:

THEOREM 1.1. 1. There is a compact strictly convex hypersurface that loses its convexity under the flow (1).

2. There is a compact and embedded hypersurface that loses its embeddedness under the flow (1).

The proof of Theorem 1.1 consists of two parts. First one constructs an initial surface that has nearly all the properties claimed. For the first part we will construct a convex hypersurface that is not strictly convex and show that it loses its convexity under the flow using simple local calculations. Concerning the embeddedness, we construct a surface that touches itself at precisely one point and show that it develops self intersection under the flow. In this step we will use Lemma 2.2 to show that the specific surface loses its convexity or embeddedness. This Lemma shows that for graphs the leading term of the evolution equations for the surface itself and of the evolution equation of its mean curvature is just a power of the Laplacian.

After that, we will disturb these initial surfaces and use the stability of our flow (Theorem 2.1) to derive the full result.

\section{General remarks}

First, let us recapitulate a known existence and stability result for equations of type (1).

Let $\operatorname{Imm}\left(M_{n}, \mathbb{R}^{n+1}\right)$ denote the space of smooth immersions of $M_{n}$ into $\mathbb{R}^{n+1}$. From Theorem 7.17 in [9] we get that for every $f_{0} \in \operatorname{Imm}\left(M_{n}, \mathbb{R}^{n+1}\right)$ there is a smooth, non-extendable solution $f: M_{n} \times\left[0, T\left(f_{0}\right)\right) \rightarrow \mathbb{R}^{n+1}$ of (1) with initial data $f_{0}$. For $\mathcal{V}:=\left\{(g, t) \in \operatorname{Imm}\left(M_{n}, \mathbb{R}^{n+1}\right) \times \mathbb{R}_{+}: t<T(g)\right\}$ let us define

$$
\Psi: \mathcal{V} \rightarrow \operatorname{Imm}\left(M_{n}, \mathbb{R}^{n+1}\right)
$$

by letting $\Psi\left(f_{0}, \cdot\right)$ be the unique solution of (1) with initial data $f_{0}$. A small modification of the proof of Theorem 7.17 leads to

THEOREM 2.1 (Theorem 7.17 in [9]). The function $\Psi$ is continuous with respect to the $C^{\infty}$ topology on $\operatorname{Imm}\left(M_{n}, \mathbb{R}^{n+1}\right)$ and the function $T$ is lower semicontinuous.

Let $\kappa_{1}, \ldots, \kappa_{n}$ denote the principle curvatures of an immersion $g \in C^{\infty}\left(M_{n}, \mathbb{R}^{n+1}\right)$ and let $\left\|A_{g}\right\|^{2}:=\sum_{i=1}^{n} \kappa_{i}^{2}$. It is well known that for a solution $f$ of (1), the mean curvature evolves according to

$$
\partial_{t} H_{f(\cdot, t)}=\left(\Delta_{f(\cdot, t)} V_{f(\cdot, t)}+\left\|A_{f(\cdot, t)}\right\|^{2} V_{f(\cdot, t)}\right)
$$

where

$$
V_{g}=\left(-\Delta_{g}\right)^{p} H_{g}+B\left(\nabla_{g}^{2 p-1} S_{g}, \nabla_{g}^{2 p-2} S_{g}, \ldots, S_{g}, n_{g}\right)
$$

for any embedding $g \in C^{\infty}\left(M_{n}, \mathbb{R}^{n}\right)$, (cf. [9, Theorem 3.2]). 
Let us consider the right-hand sides of the Equations (1) and (2) for the graph of a function $u$. The next lemma tells us that at points with $D u=0$ the part of highest order of these terms is simply a power of the Laplacian on $\mathbb{R}^{n}$.

LEMMA 2.2. There are smooth functions $\tilde{B}$ and $\tilde{\tilde{B}}$ with the following property: For $u \in C^{\infty}(U, \mathbb{R}), U \subset \mathbb{R}^{n}$ open, $x_{0} \in U$ with $D u\left(x_{0}\right)=0$ we set

$$
\begin{aligned}
f: U & \rightarrow \mathbb{R}^{n+1} \\
f(z) & =(z, u(z))
\end{aligned}
$$

and take the mean curvature with respect to the upward pointing unit normal $v_{f}=$ $(-\nabla u, 1) / \sqrt{1+\|\nabla u\|^{2}}$. Then,

$$
\left.V_{f}\right|_{\left(x_{0}, u\left(x_{0}\right)\right)}=-\left(-\Delta_{\mathbb{R}^{n}}\right)^{p+1} u\left(x_{0}\right)+\tilde{B}\left(D^{2 p+1} u\left(x_{0}, \ldots, D u\left(x_{0}\right), u\left(x_{0}\right)\right)\right.
$$

and

$$
\begin{aligned}
& \left.\left(\Delta_{f} V_{f}+\left\|A_{f}\right\|^{2} V\right)\right|_{x_{0}, u\left(x_{0}\right)} \\
& \quad=\left(-\Delta_{\mathbb{R}^{n}}\right)^{p+2} u\left(x_{0}\right)+\tilde{\tilde{B}}\left(D^{2 p+3} u\left(x_{0}\right), \ldots, D u\left(x_{0}\right), u\left(x_{0}\right)\right) .
\end{aligned}
$$

Proof. In this case, the first fundamental form can be expressed by $g_{i j}=\delta_{i j}+\partial_{i} u \partial_{j} u$ and the second fundamental form with respect to the upward pointing unit normal

$$
v_{f}=(-\nabla u, 1) / \sqrt{1+\|\nabla u\|^{2}}
$$

reads

$$
h_{i j}=\frac{\partial_{i j} u}{\sqrt{1+\|\nabla u\|^{2}}} .
$$

The Laplace-Beltrami operator is known to be

$$
\Delta_{f}=\frac{1}{\sqrt{g}} \partial_{i}\left(\sqrt{g} g^{i j} \partial_{j}\right)
$$

Together with the product rule one gets

$$
V=(-1)^{p} g^{i_{1} j_{1}}, \ldots, g^{i_{p} j_{p}} \partial_{i_{1}, j_{1}, \ldots, i_{p+1}, j_{p+1}} u+\tilde{B}\left(D^{2 p+1} u, D^{2 p} u, \ldots, u\right)
$$

and

$$
\begin{aligned}
& \Delta V+\|A\|^{2} V \\
& \quad=(-1)^{p} g^{i_{1} j_{1}}, \ldots, g^{i_{p+1} j_{p+2}} \partial_{i_{1}, j_{1}, \ldots, i_{p+2}, j_{p+2}} u+\tilde{B}\left(D^{2 p+1} u, D^{2 p} u, \ldots, u\right) .
\end{aligned}
$$

Using $\left.g_{i j}\right|_{x_{0}, u\left(x_{0}\right)}=\delta_{i j}$, the claim follows. 


\section{Loss of embeddedness}

We start by constructing a family of immersions $f_{\alpha}$ such that $f_{\alpha}$ are embeddings for all $\alpha>0$ and $f_{0}$ develops a selfintersection under the flow (1). We will achieve the latter constructing $f_{0}$ in such a way that it contains parts of the graphs of the functions

$$
\begin{aligned}
u_{M}: \mathbb{R}^{n} & \rightarrow \mathbb{R} \\
u_{M}(x) & :=(-1)^{p+1} M\|x\|^{2(p+1)}+\|x\|^{2 p}
\end{aligned}
$$

and $-u_{M}$, that lie in a small ball around the origin. Using Lemma 2.2, we will see that $f_{0}$ develops selfintersections if $M$ is big enough. Note that $u_{M}>0$ on $B_{\frac{1}{\sqrt{M}}}(0)-\{0\}$.

In the following, $\mathbb{S}^{n}$ is always considered as the subset $\left\{x \in \mathbb{R}^{n+1} \mid\|x\|=1\right\}$.

LEMMA 3.1. For all $M>1$ there is a continuous family of smooth immersions $f_{\alpha}: \mathbb{S}^{n} \rightarrow \mathbb{R}^{n+1}, \alpha \in[0,1]$ such that

1. For all $\alpha>0, f_{\alpha}$ is an embedding.

2. There are two disjoint topological discs $D_{-}, D_{+} \subset \mathbb{S}^{n}$ with

$$
f_{0}\left(D_{+}\right)=\operatorname{graph}\left(\left.u_{M}\right|_{\frac{1}{2 \sqrt{M}}^{(0)}}\right)
$$

and

$$
f_{0}\left(D_{-}\right)=\operatorname{graph}\left(-\left.u_{M}\right|_{B_{\frac{1}{2 \sqrt{M}}}}(0)\right)
$$

Proof. We choose a smooth cutoff function $\phi \in C^{\infty}\left(\mathbb{R}^{n},[0,1]\right)$ with $\phi=1$ on $B_{\frac{1}{2 \sqrt{M}}}(0)$ and spt $\phi \subset \subset B_{\frac{1}{\sqrt{M}}}(0)$. Furthermore, let $\Pi: \mathbb{R}^{n+1} \rightarrow \mathbb{R}^{n}$ be defined by $\Pi\left(x_{1}, \ldots, x_{n+1}\right):=\left(x_{1}, \ldots, x_{n}\right)$. We then set

$$
f_{\alpha}(p):=\left\{\begin{array}{cc}
\left(\begin{array}{c}
\Pi(p) \\
\left.(1-\phi(p)) \cdot \sqrt{1-\|\Pi(p)\|^{2}}+\phi(p) \cdot\left(u_{M}(\Pi(p))+\alpha\right)\right)
\end{array}\right) & \text { if } p_{n+1} \geq 0 \\
\left(\begin{array}{c}
\Pi(p) \\
\left.-(1-\phi(p)) \cdot \sqrt{1-\|\Pi(p)\|^{2}}-\phi(p) \cdot\left(u_{M}(\Pi(p))+\alpha\right)\right)
\end{array}\right) & \text { else }
\end{array}\right.
$$

and see that this defines a family with the properties claimed.

Proof of the first part of Theorem 1.1. Let $f_{\alpha}$ be the family of immersion from Lemma 3.1 and let $p_{-} \in D_{-}, p_{+} \in D_{+}$be such that $f_{0}\left(p_{-}\right)=0=f_{0}\left(p_{+}\right)$. Furthermore, let $T: \mathcal{V} \rightarrow(0, \infty]$ be the time of existence and $\Psi$ be the semiflow of Theorem 2.1. We then have by Lemma 2.2

$$
\begin{aligned}
\left.\frac{\mathrm{d}}{\mathrm{d} t} \Psi\left(f_{0}, t\right)\left(p_{+}\right)\right|_{t=0} & =-\left.\frac{\mathrm{d}}{\mathrm{d} t} \Psi\left(f_{0}, t\right)\left(p_{-}\right)\right|_{t=0} \\
& =-M n^{p+1}(2 p+2) !+\tilde{B}(0,(2 p+1) !, 0, \ldots, 0)<0
\end{aligned}
$$


if $M$ is large enough. Using the shape of $f_{0}$ and the continuity of $\Psi\left(f_{0}, \cdot\right)$, this implies that there is a small $\delta_{0}>0$ such that

$$
\begin{array}{r}
\inf _{p \in \partial D_{+}}\left\langle\Psi\left(f_{0}, \delta_{0}\right)(p), e_{n+1}\right\rangle>0, \\
\sup _{p \in \partial D_{-}}\left\langle\Psi\left(f_{0}, \delta_{0}\right)(p), e_{n+1}\right\rangle<0, \\
\left\langle\Psi\left(f_{0}, \delta_{0}\right)\left(p_{+}\right), e_{n+1}\right\rangle<0,
\end{array}
$$

and

$$
\left\langle\Psi\left(f_{0}, \delta_{0}\right)\left(p_{-}\right), e_{n+1}\right\rangle>0 .
$$

Since $T$ is lower semicontinuous, and since the function $\alpha \rightarrow \Psi\left(f_{\alpha}, \delta_{0}\right)$ is continuous, there is an $\alpha_{0}>0$ such that

$$
\begin{array}{r}
\inf _{p \in \partial D_{+}}\left\langle\Psi\left(f_{\alpha}, \delta_{0}\right)(p), e_{n+1}\right\rangle>0, \\
\sup _{p \in \partial D_{-}}\left\langle\Psi\left(f_{\alpha}, \delta_{0}\right)(p), e_{n+1}\right\rangle<0, \\
\left\langle\Psi\left(f_{\alpha}, \delta_{0}\right)\left(p_{+}\right), e_{n+1}\right\rangle<0,
\end{array}
$$

and

$$
\left\langle\Psi\left(f_{\alpha}, \delta_{0}\right)\left(p_{-}\right), e_{n+1}\right\rangle>0
$$

for all $0 \leq \alpha \leq \alpha_{0}$. Let $0<\alpha<\alpha_{0}$. Then, $f_{\alpha}$ is an embedding and the above relations imply that for all $0<\alpha<\alpha_{0}$ there are $\tilde{p}_{-} \in D_{-}$and $\tilde{p}_{+} \in D_{+}$with $\Psi\left(f_{\alpha}, \delta_{0}\right)\left(p_{+}\right)=\Psi\left(f_{\alpha}, \delta\right)\left(p_{-}\right)$. Hence $\Psi\left(f_{\alpha}, \delta_{0}\right)$ is not embedded which proves the theorem.

\section{Loss of convexity}

To show that the surfaces can lose convexity as well, we construct an initial surface by cutting a small hole out of a sphere and fill it with a small part of the graph of the function

$$
\begin{aligned}
& u_{M}: \mathbb{R}^{n} \rightarrow \mathbb{R}^{n+1} \\
& x \rightarrow M(-1)^{p+1}\|x\|^{2 p+4}+\|x\|^{2 p+2}
\end{aligned}
$$

in such a way that we get a convex $C^{1,1}$ hypersurface. Lemma 2.2 and Equation (2) show that

$$
\partial_{t} H=-M n^{(p+2)}(2 p+4) !+\tilde{\tilde{B}}(0,(2 p+2) !, 0, \ldots, 0)<0
$$

if $M$ is large enough. As $H=0$ in 0 , this will imply that the surface loses its convexity. We set $\tilde{M}:=M \frac{(2 p+4)(2 p+3)}{(2 p+2)(2 p+1)}$ and note that $u_{M}$ is strictly convex on $B_{\frac{1}{\sqrt{\tilde{M}}}}(0)$.

Let us make this construction more precise. 
LEMMA 4.1. For all $M>1$ there is a smooth convex embedding $f_{0}: \mathbb{S}^{n} \rightarrow \mathbb{R}^{n+1}$ such that there is a topological open disc $D \subset \mathbb{S}^{n}$ such that

$$
f_{0}(D)=\operatorname{graph}\left(\left.u_{M}\right|_{\frac{1}{2 \sqrt{\tilde{M}}}}(0)\right) .
$$

Proof. Let $\tilde{u}_{M}: \mathbb{R} \rightarrow \mathbb{R}$ be defined by $\tilde{u}_{M}(r):=M(-1)^{p+1} r^{2 p+4}+r^{2 p+2}$. For $a:=\tilde{M}^{-1 / 2} / \tilde{u}^{\prime}\left(\tilde{M}^{-1 / 2}\right)+\tilde{u}\left(\tilde{M}^{-1 / 2}\right), R:=\sqrt{\left(a-\tilde{u}\left(\tilde{M}^{-1 / 2}\right)\right)^{2}+1 / \tilde{M}}$ we set

$$
\hat{u}_{M}(r):= \begin{cases}\tilde{u}(r) & \text { if }|r| \leq 1 / \sqrt{\tilde{M}} \\ a-\sqrt{R^{2}-\|r\|^{2}} & \text { else. }\end{cases}
$$

The choice of $R$ and $a$ guarantees $\hat{u}_{M} \in C_{\mathrm{loc}}^{1,1}(-R, R) \cap C^{\infty}((-R, R) /\{ \pm 1 / \sqrt{\tilde{M}}\})$ and is strictly convex on $(-R, R) /\{ \pm 1 / \sqrt{\tilde{M}}\}$. Hence, due to the result of Ghomi in [10] there is a convex function $\tilde{\tilde{u}}_{M} \in C^{\infty}((-R, R))$ such that

$$
\hat{u}_{M}=\tilde{\tilde{u}}_{M} \quad \text { on } \quad(-R, R) /\left(B_{\varepsilon}\left(-\tilde{M}^{-1 / 2}\right) \cup B_{\varepsilon}\left(\tilde{M}^{-1 / 2}\right)\right)
$$

where $\left.\varepsilon=\frac{1}{2} \min \left\{\tilde{M}^{-1 / 2}, R-\tilde{M}\right)^{-1 / 2}\right\}$. We set

$$
f_{0}(p):= \begin{cases}\left(\begin{array}{c}
R \Pi(p) \\
\left.\tilde{u}_{M}(R \Pi(p))\right)-a
\end{array}\right) & \text { if } p_{n+1} \leq 0 \\
R p+R e_{n+1} & \text { else. }\end{cases}
$$

LEMMA 4.2. For all $M>1$ there is a smooth family of embeddings $f_{\alpha}: \mathbb{S}^{n} \rightarrow \mathbb{R}^{3}$, $\alpha \in[0,1]$, such that

1. For all $\alpha>0, f_{\alpha}$ is strictly convex.

2. There is a topological disc $D \subset \mathbb{S}^{2}$ and an $\epsilon>0$ with

$$
f_{0}(D)=\operatorname{graph}\left(\left.u_{M}\right|_{B_{\frac{1}{2 \sqrt{M}}}}(0)\right) .
$$

Proof. Let $\tilde{f}_{t}, t \in[0, T)$ be a solution of the mean curvature flow with initial data $f_{0}$ from Lemma 4.2. Since $f_{0}$ is convex, it is well known that $f_{t}$ is strictly convex for all $0<t<T$. We finish the proof by setting $f_{\alpha}:=\tilde{f}_{\alpha T / 2}$.

Proof of the second part of Theorem 1.1. Let $f_{\alpha}$ be the family of embeddings from Lemma 4.2 and $T, \Psi$ be as in the statement of Theorem 2.1. Furthermore, let $p \in D$ be such that $f_{0}(p)=0$. Then, Equation (7) implies that $\frac{\mathrm{d}}{\mathrm{d} t} H_{f_{0}}<0$ if $M$ is large enough. Together with $H_{f_{0}}=0$, we then obtain $H_{\psi\left(f_{0}, \delta_{0}\right)}<0$ for some $\delta_{0}<T\left(f_{0}\right)$. Since $T$ is lower semicontinuous, and since the function $\alpha \rightarrow \Psi\left(f_{\alpha}, \delta_{0}\right)$ is continuous, there is an $\alpha_{0}>0$ such that

$$
H_{\psi\left(f_{\alpha}, \delta_{0}\right)}<0
$$


for all $0 \leq \alpha<\alpha_{0}$. Hence, all the strictly convex surfaces $f_{\alpha}, 0<\alpha<\alpha_{0}$ lose their convexity under the evolution equation (1).

\title{
REFERENCES
}

[1] Ben Andrews. Contraction of convex hypersurfaces in Euclidean space. Calc. Var. Partial Differential Equations, 2(2):151-171, 1994.

[2] Michael Gage and Richard S. Hamilton. The heat equation shrinking convex plane curves. J. Differential Geom., 23(1):69-96, 1986.

[3] Gerhard Huisken. Flow by mean curvature of convex surfaces into spheres. J. Differential Geom., 20(1):237-266, 1984.

[4] Yoshikazu Giga and Kazuo Ito. Loss of convexity of simple closed curves moved by surface diffusion. In Topics in nonlinear analysis, volume 35 of Progr. Nonlinear Differential Equations Appl., pages 305-320. Birkhäuser, Basel, 1999.

[5] Uwe F. Mayer and Gieri Simonett. Self-intersections for the surface diffusion and the volume-preserving mean curvature flow. Differential Integral Equations, 13(7-9):1189-1199, 2000.

[6] Kazuo Ito. The surface diffusion flow equation does not preserve the convexity. Sūrikaisekikenkyūsho Kōkyūroku, (1105):10-21, 1999. Nonlinear evolution equations and applications (Japanese) (Kyoto, 1998).

[7] Uwe F. Mayer and Gieri Simonett. Self-intersections for Willmore flow. In Evolution equations: applications to physics, industry, life sciences and economics (Levico Terme, 2000), volume 55 of Progr. Nonlinear Differential Equations Appl., pages 341-348. Birkhäuser, Basel, 2003.

[8] Uwe F. Mayer and Gieri Simonett. A numerical scheme for axisymmetric solutions of curvaturedriven free boundary problems, with applications to the Willmore flow. Interfaces Free Bound., 4(1):89-109, 2002.

[9] Gerhard Huisken and Alexander Polden. Geometric evolution equations for hypersurfaces. In Calculus of variations and geometric evolution problems (Cetraro, 1996), volume 1713 of Lecture Notes in Math., pages 45-84. Springer, Berlin, 1999.

[10] Mohammad Ghomi. The problem of optimal smoothing for convex functions. Proc. Amer. Math. Soc., 130(8):2255-2259 (electronic), 2002.

\author{
S. Blatt \\ Albert-Einstein Institut, \\ Am Mühlenberg 1, \\ 14476 Postdam-Golm, Germany \\ E-mail: simon.blatt@aei.mpg.de
}

